\title{
Thanks to JMCP Peer Reviewers, 2015
}

The Journal of Managed Care \& Specialty Pharmacy would like to thank the 366 reviewers who have reviewed manuscripts in 2015 and contributed to the high quality of articles in JMCP. Without this excellent level of reviewer involvement, $J M C P$ would not be one of the most respected journals in health care services, connecting authors and innovators to accelerate advances in managed care pharmacy.

\section{—Laura E. Happe, PharmD, MPH Editor-in-Chief}

Ibrahim Abbass, RPh, MS, PhD

Mousa Abkhezr, PharmD

Paul N. Abourjaily, PharmD, BCPS

Safiya Abouzaid, PharmD, MPH

Susan Abughosh, PhD

Daphne T. Adelman, BSN, MBA

Sarah Elaine Adkins, BS, RPh, PharmD

Parul Agarwal, MPH

Patricia Melo Aguiar, PhD

Vishal Ahuja, PhD

Mayank Ajmera, PhD

Medinat S. Akinbi, PharmD

Nicole Paolini Albanese, PharmD, CDE, BCACP

Matthew Alcusky, PharmD, MS

Ebtihag Owayed Alenzi, BPharm

J. Daniel Allen, PharmD

Aylin E. Altan, PhD

Miriam Alvarez-Payero, MS

Abdalla Aly, PhD

Sheta Ara, PharmD

Carl V. Asche, PhD

Kirsten Axelsen-Carmel, MS

Jay P. Bae, PhD

Christine Leigh Bagley, PharmD

Danial E. Baker, BPharm, PharmD

William L. Baker, PharmD

Vishal Bali, PhD

John J. Barron, PharmD

Dea T. Belazi, PharmD, MPH, PAHM

Christopher Bell, MS

Brandon K. Bellows, PharmD

Sandra Benavides, PharmD

Todd Berner, MD

Gary Besinque, PharmD, FCSHP

Maribeth M. Bettarelli, PharmD, RPh

Boyang Bian, MS

Sarah J. Billups, PharmD

Joseph Biskupiak, PhD, MBA
Sandra G. Blake, PhD, MBA

Crystal Blankenship, PharmD

Steven Blume, MS

Susan C. Bolge, PhD

T. Christopher Bond, PhD

Mary Ellen Bonk, PharmD

Jeffrey S. Borer, MD

Jonathan R. Bouchard, MS, RPh

Thomas J. Bramley, PhD, RPh

Diana Brixner, PhD

Carolyn Brown, PhD

Courtney Rhiannon Brown, PharmD

Dana N. Brown, BS, PharmD

Jean Brown, RPh, PharmD

Joshua David Brown, PharmD, MS

Mark N. Brueckl, BSPharm, MBA

Amanda Brummel, PharmD

Crystal J. Bryner, RPh, MBA

Ami Buikema, MPH

Elizabeth Marie Sutton Burke, PharmD

Sharon Burks, PharmD

Julie N. Burris, PharmD

Shobhna Butler, PharmD, MBA

Stacey Dacosta Byfield, PhD, MPH

Elizabeth Jane Campagna, MS

William Canestaro, MSc

Mary Jo Carden, RPh, JD

Karen Carroll, PharmD

Julian Casciano, BS

Jamie J. Cavanaugh, PharmD, CPP, BCPS

Laurie Michelle Cavendish, PharmD

Tim M. Cernohous, PharmD

James Chan, PharmD, PhD

Jongwha Chang, $\mathrm{PhD}$

Kristen Dee Charlson, PharmD

Satabdi Chatterjee, MS, PhD

T. Craig Cheetham, PharmD, MS

Lung-I Cheng, PhD
Donna Chiefari, BScPharm, PharmD

Chanadda Chinthammit, BSPharm, MS

Viktor Chirikov, MS

Shelley Chun, PharmD

Melanie Claborn, PharmD

Todd Cooperman, PharmD, MBA

Kenny Correia, PharmD

Joseph Edward Couto, PharmD

Joyce Cramer, BS

Eric J. Culley, PharmD, MBA

Akash Dandappanavar, PharmD, MPH, MA

Theodore Darkow, PharmD

Estella Davis, PharmD, BCPS

Sabina DeGeest, PhD

Melissa Suzanne Denno, PharmD

Pooja R. Desai, MS

Kelli Devlin, BSPharm

Amol Dhamane, BPharm, MS

Thomas J. Dilworth, PharmD

Qian Ding, PhD

Alexander James Dong, PharmD

Yana Alesa Doughty, PharmD

Anna 0. D'Souza, PhD

Michael Durkin, MS

Nilanjana Dwibedi, MBA, MS, PhD

Joseph Ted Dye, PhD, RPh

Michael T. Eaddy, PharmD, PhD

Lisa A. Edwards, PharmD, RPh

Maguy El Hajj, PharmD, BCPS

Jeffrey J. Ellis, PharmD, MS

Derek Ems, MPH

Sara Carruth Erickson, PharmD

Steven R. Erickson, PharmD

Dominick Esposito, PhD

Joel F. Farley, PhD

Kevin C. Farmer, PhD

Steven R. Feldman, MD, PhD

Beckie A. Fenrick, PharmD 


\section{Thanks to JMCP Peer Reviewers, 2015 (continued)}

Stanley E. Ferrell, BSPharm

Bithia Fikru, PharmD, MPA

Leslie S. Fish, PharmD

Karen Fitzner, PhD

Renee R. Fleming, RPh, MBA

Sara Forrester, PharmD, MS

Philip Foulis, MD, MPH

Jessica Frank, PharmD

Dustin D. French, PhD

Joshua J. Gagne, PharmD, ScD

Pranav K. Gandhi, PhD, MS, BPharm

Xin Gao, PhD

Maria M. Garcia, MD, MPH, FACP

Lou Garrison, PhD

Justin Gatwood, PhD, MPH

Rodney Gedey, PharmD

David A. Gettman, PhD, MBA, BSPharm

Teresa Gibson, PhD

Monika Gil, PharmD

Aggie Gilbert, RN

Jasjit Gill, BSPharm, PharmD

Brianne Giordano, PharmD

Kimberly Gittings, PharmD (candidate)

Patrick P. Gleason, PharmD, BCPS

Boris Gorsh, PharmD

Phyllis A. Graham, BSPharm

Bonnie Greenwood, PharmD

Scott Karr Griggs, PharmD, PhD

Matthew C. Grissinger, BSPharm

Paul 0. Gubbins, PharmD

Komal R. Gupte-Singh, BPharm, MS

Anna M. Hall, PharmD

Rachel Halpern, PhD, MPH

Cheryl S. Hankin, PhD

Richard A. Hansen, PhD

Ryan N. Hansen, PhD, PharmD

Philip Hansten, PharmD

Mohamed G. Hashem, PharmD

Noelle K. Hasson, PharmD

Joel W. Hay, PhD

Lindy Haynes, PharmD

Pamela C. Heaton, PhD, RPh

Gregory Hess, MD, MBA, MSc

Ana Hincapie, PhD

Jan D. Hirsch, RPh, PhD
Mei Jen Ho, PharmD, MSPH

Michelle Lynn Holbrook, PharmD

David A. Holdford, PhD

Andrew Howe, PharmD

Rita L. Hui, PharmD, MS

Jessica Hull, PharmD, BCPS

Anna Hung, PharmD

Reethi Narasimhan lyengar, PhD, MBA, MHM

Mark Andrew Jackson, BScPharm, BComm, $\mathrm{RPh}$

Paul Jeffrey, PharmD

Ruixuan Jiang, BS

Shan Jiang, PhD

Yawen Jiang, MS

Tianze Jiao, PhD

Shu Jing, PharmD

Deborah Taira Juarez, ScD

Kyoungrae (Jeah) Jung, PhD

Abby A. Kahaleh, BPharm, MS, PhD, MPH

Pravin Shivaji Kamble, BPharm, MTech, RPh, MS, PhD

Abir 0. Kanaan, PharmD

Shellie L. Keast, PharmD, PhD

Elizabeth Maureen Kelly, PharmD

James Kenney, BS, MBA

David M. Kern, MS

Michael H. Kim, MD

Robert Klein, MS

Donald G. Klepser, PhD, MBA

Katherine K. Knapp, PhD

Casey Ryan Koch, PharmD

Stephen J. Kogut, MBA, PhD

Jennifer S. Korsnes, MS

Pamela Landsman-Blumberg, DrPH

Daniel C. Lane, PharmD, PhD

Julie C. Lauffenburger, PharmD, PhD

Janet Lee, PhD

Jeff Lee, PharmD, FCCP

Peter J. Lee, PharmD, MBA

YoonJung Lee, PharmD

Julian Talbot Leland, BS

Matthew David Lennertz, PharmD

Kimberly Lenz, PharmD

Christine Leong, PharmD

Charles C. Lewis, BSPharm, MBA
Haitao Li, PharmD, MS

Joshua N. Liberman, PhD

Alexandra Tungol Lin, PharmD

Jay Lin, PhD, MBA

Vincent Lin, PharmD, MS

You-Li Ling, MS

Melissa Lingohr-Smith, PhD

Tasneem Lokhandwala, PhD

Kajua Betsy Lor, PharmD

Rex S. Lott, PharmD

Gregory A. Low, BPharm, PhD

Heidi Renee Luder, PharmD, MS, BCACP

Alan Lyles, MPH, ScD, BSPharm, BA

Tatiana Makhinova, MS

Daniel C. Malone, PhD

Carolyn Martin, MSW

Aman Deep Matharoo, PharmD

Josephine A. Mauskopf, PhD

Anthony J. May, PharmD, MBA

Milena McLaughlin, PharmD, MSc

Trent McLaughlin, PhD

Darshan Mehta, MS

Hemalkumar B. Mehta, MS, PhD

Candice Mercadel, PharmD

Andrew Merker, PharmD

Rick Miller, RPh, MBA, CSP

Leticia Moczygemba, PharmD, PhD

Daniela Claudia Moga, MD, PhD

Kim Moon, PharmD

Radhika Nair, PhD

David Paul Nau, PhD

Robert P. Navarro, PharmD

Uche Anadu Ndefo, PharmD

Erin Neal, PharmD

Peter J. Neumann, ScD

Brand Newland, PharmD

Phung Kim Nguyen, PharmD

Candace M. Nichols, PharmD, BCPS

James Notaro, RPh, PhD

Heather Nyman, PharmD

Kevin Odell, PharmD

Richard L. Ogletree, Jr., PharmD

Robert L. Ohsfeldt, PhD

Arinze Nkemdirim Okere, PharmD, MS, BCPS

Alexander M. Orr, PharmD 


\section{Thanks to JMCP Peer Reviewers, 2015 (continued)}

Terrence 0'Shea, PharmD

Gary Owens, MD

Francis B. Palumbo, PhD

Xiaoyun Pan, MS, PhD

Sharon K. Park, PharmD

Margaret K. Pasquale, PhD

Mark E. Patterson, PhD, MPH

Michael Pazirandeh, PharmD

Patricia J. Peeples, PhD, MS, RPh

Peter M. Penna, PharmD

Jason Perepelkin, BA, BComm, MSc, PhD

Andrew M. Peterson, PharmD

Sarah Priddy, PhD

Janice Pringle, $\mathrm{PhD}$

Daryl Pritchard, PhD

Lauren Pusateri, PharmD

Bruce S. Pyenson, FSA, MAAA

Nabeel Qureshi, PharmD, MPH

Aditya Raju, BPharm, MS

Ambili Ramachandran, MD, MSc

Kate L. Ramsay, PharmD

Rafia S. Rasu, PhD

Kali Raval, PharmD

Prabashni Reddy, PharmD

Claiborne (Gene) E. Reeder, PhD

Lori Reisner, PharmD

Jinma Ren, PhD

Nathaniel James Rhodes, PharmD

Sheldon J. Rich, RPh, PhD

Cathlene Richmond, PharmD, BCPS

Nathaniel Marc Rickles, PharmD, PhD

Andrew W. Roberts, PharmD

Carly Rodriguez, PharmD

M. Christopher Roebuck, MBA, PhD

Matthew Romo, PharmD

Matthew D. Rousculp, PhD, MPH

Deanna M. Rowe, PharmD

Elan Rubinstein, PharmD, MPH

Naomi C. Sacks, PhD

Qayyim Said, PhD

Prashant Sakharkar, PharmD, MPH

Brian Christopher Sauer, PhD

Kimberly Saverno, PhD, RPh

Kunal Saxena, MS

Vernon F. Schabert, PhD
Caroline Schaefer, MBA

Jeff Schein, DrPH, MPH

Kathy Schulman, MA

Michael Serbin, BS, PharmD (candidate)

Mark S. Shaefer, PharmD

Manan Shah, PharmD, PhD

Krishna P. Sharma, PhD

Fadia T. Shaya, PhD, MPH

Natalia G. Shcherbakova, PhD

Xian Shen, MS

Christina H. Sherrill, PharmD

Katarzyna Shields, PharmD, MBA

Kathleen Shoemaker, PharmD, MBA

Martha Shumway, $\mathrm{PhD}$

Matthew A. Silva, PharmD, RPh, BCPS

Jonathan Silver, MD, MS, MBA

Jason C. Simeone, PhD

Sonal Singh, MD, MPH

Devada Singh-Franco, PharmD

Grant H. Skrepnek, PhD

Julia Frances Slejko, PhD

Mary Buatti Small, MS

Alesha J. Smith, PhD

Timothy W. Smith, BA

Scott A. Soefje, PharmD, MBA

Richard H. Stanford, PharmD, MS

Marilyn R. Stebbins, PharmD

David Stenehjem, PharmD

Jennifer M. Stephens, PharmD

Craig Stephen Stern, RPh, PharmD, MBA

Debbie J. Stern, RPh

Debora Sternaman, BS, PharmD

James G. Stevenson, PharmD, FASHP

Eileen M. Stock, PhD

Samuel Stolpe, PharmD

Linda L. Sturm, MHA, RPh, BCPS

Wenqing Su, MS

Kent Summers, PhD

Ryan Swanson, PharmD

Patty Taddei-Allen, PharmD

Sandra E. Talbird, MSPH

Xi Tan, PharmD

Reginald Tayaben, PharmD

Ann M. Taylor, MPH, MCHES

Daniel Mark Tomaszewski, PharmD, PhD
Josephine (Nhu) Tran, PharmD, MS

Luke S. Tso, PharmD

Ozgur Tunceli, PhD

Robin Turpin, PhD

Connie A. Valdez, PharmD, MSEd, BCPS

Preeti Vallabh, PharmD

Job F.M. van Boven, PharmD, PhD

Julie C. Jacobson Vann, PhD, MS, RN

Tien Vuong, PharmD

Rolin Wade, RPh, MS

Linda Timm Wagner, PharmD

Surrey M. Walton, PhD

Melea A. Ward, PharmD, MS

Albert I. Wertheimer, BS, MBA, PhD

Laurie Wesolowicz, PharmD

Sarah M. Westberg, PharmD

Ria Westergaard, PharmD

Craig White, BE, PhD (candidate)

Marnie Wickizer, PharmD

Vincent J. Willey, PharmD

James P. Wilson, BSc, PharmD, MSc, PhD

Amanda Winters, PharmD

Matthew J. Witry, PharmD, PhD

Mary Claire Wohletz, PharmD

Karen Worley, $\mathrm{PhD}$

Chengxiang $\mathrm{Wu}, \mathrm{PhD}$

Chung-Hsuen Wu, PhD

Joanne Wu, MD, MS

Jun $\mathrm{Wu}, \mathrm{PhD}$

Zhimin Xiao, MD, MS, MPH

Jing Xie, MD, MPH, ScD

Hongbo Yang, PhD

Winnie M. Yang, PharmD

Xiaoqin Yang, $\mathrm{PhD}$

Kai Yeung, PharmD, MS

Peinie Penny Young, PharmD

Elaine Yu, PharmD

Junhua Yu, PhD/MS

Jing Yuan, BPharm

Barbara Jeanne Zarowitz, PharmD, CGP,

BPS, FCCP

Yongming Zhao, PhD

Yichen Zhong, MHS

Zheng-Yi Zhou, PhD

Leah L. Zullig, PhD, MPH 